Voix et Images

voixetimages

\title{
Notre premier auteur comique : Pierre Petitclair (1813-1860)
}

\section{Jean-Claude Noël}

Volume 6, numéro 1, automne 1980

Gilles Marcotte

URI : https://id.erudit.org/iderudit/200253ar

DOI : https://doi.org/10.7202/200253ar

Aller au sommaire du numéro

Éditeur(s)

Les Presses de l'Université du Québec

ISSN

0318-9201 (imprimé)

1705-933X (numérique)

Découvrir la revue

Citer cet article

Noël, J.-C. (1980). Notre premier auteur comique : Pierre Petitclair (1813-1860).

Voix et Images, 6(1), 117-126. https://doi.org/10.7202/200253ar d'utilisation que vous pouvez consulter en ligne.

https://apropos.erudit.org/fr/usagers/politique-dutilisation/ 


\title{
Notre premier auteur comique : Pierre Petitclair (1813-1860)
}

\author{
par Jean-Claude Noël
}

Pierre Petitclair est le premier qui, né au Canada, a écrit pour le théâtre. C'est pourquoi il a le privilège de voir son nom figurer dans la plupart de nos manuels d'histoire littéraire. Cependant, la gloire de ce titre n'a pas suscité l'intérêt des chercheurs et, jusqu'à maintenant, les mieux renseignés se bornent à donner la liste de ses ouvrages ou à en résumer les plus importants. Certes, Petitclair n'a pas produit de chef-d'œuvre et ses écrits déçoivent sur le plan littéraire. Mais à ce compte, combien de nos écrivains du siècle passé méritent de retenir notre attention? Si on les lit encore aujourd'hui, c'est généralement parce qu'ils témoignent d'une époque et d'une civilisation où nous devons chercher l'explication de notre identité et de nos traditions. L'œuvre de Petitclair offre pareil témoignage. Le sociologue y trouvera sûrement des indications valables et il n'est pas dit que, pour sa part, le critique littéraire n'y verra pas quelque mérite ou même quelque originalité. Aussi convient-il de savoir qui est Pierre Petitclair et de se demander quel est le sens de son œuvre.

La vie de Petitclair est mal connue et tout au plus peut-on en indiquer les grandes lignes. Le détail manque, et ce n'est pas sans raison. Timide au point de paraître farouche et indomptable, Petitclair répugnait à se livrer. Aussi la seule biographie que nous ait laissée de lui un de ses contemporains, Louis-Michel Darveau, est-elle incomplète et contient-elle plusieurs inexactitudes ${ }^{1}$. De plus, Petitclair est allé, à partir de 1837, habiter sur la Côte Nord, se déplaçant sans cesse et ne laissant derrière lui presque aucune trace. Les rares documents trouvés sont peu révélateurs ou lancent sur des pistes qui aboutissent fatalement à des impasses. Le chercheur en est donc réduit à des conjectures, à des suppositions. Petitclair chérissait la solitude. C'était très certainement une disposition de son caractère et c'était peut-être aussi, de sa part, une juste clairvoyance, car, depuis sa mort, peu de gens ont tenté d'éclairer son destin. Résigné à l'oubli, notre premier auteur de théâtre semble s'y complaire encore maintenant.

Pierre Petitclair est né à Saint-Augustin de Portneuf, le 12 octobre $1813^{2}$. Dès le lendemain, son père, prénommé Pierre aussi, le fit baptiser à la nouvelle église du village. Le prêtre, un certain abbé Antoine Lefrançois, dressa l'acte comme suit : 
Le treize octobre mil huit cent treize, par moi, prêtre soussigné, a été baptisé Pierre, né la veille du dit jour du légitime Mariage de Pierre Petit-Clair, cultivateur de l'Ancienne Lorette, résidant à SaintAugustin, et de Cécile Moisan. Parrain Jean-Baptiste Hamel; marraine Magdeleine Bussière Moisan qui tous ont déclaré ne savoir signer ${ }^{3}$.

L'abbé, note-t-on, a écrit Petit-Clair, mais Pierre, l'écrivain, préférera orthographier son nom d'un seul trait. Aussi est-ce à tort que certains historiens littéraires emploient l'homonyme Petitclerc pour parler de lui. If est vrai toutefois que le premier ancêtre de la famille à venir au Canada écrivait son nom de cette manière.

Cet ancêtre, Pierre Petitclerc, fils de Jean Petitclerc et de Marie Puize, de Saint-Cénery de Pleumartin, dans l'ancienne province du Poitou, vint en Nouvelle-France vers 1670. S'étant établi à Québec, il y épousa, le 11 septembre 1673, Françoise Paris, une Champenoise de Saint-Pierre de Sens. Leurs descendants se dispersèrent dans les villages environnant Québec, surtout à l'Ancienne Lorette et à Saint-Augustin, où ils s'adonnèrent à l'agriculture 4 .

A la quatrième génération, Pierre, le père de l'écrivain, revint habiter Québec. Né à l'Ancienne Lorette, il y épousa Cécile Moisan, le 20 octobre $1812^{5}$, et tous deux allèrent demeurer à Saint-Augustin où naquirent leurs deux seuls enfants : Pierre en 1813 et Marie-Cécile en 1815. Après, la famille se retrouvait à nouveau à Québec au moment où le jeune Pierre atteignait l'âge scolaire.

L'enfant fut d'abord inscrit à une des écoles fondées par JosephFrançois Perrault, faubourg Saint-Louis. Là, il apprit la lecture, l'écriture, le calcul, l'anglais et la grammaire française ${ }^{6}$. Parmi ses confrères se trouvait celui qui allait devenir notre historien national. De quatre ans plus âgé que Petitclair, François-Xavier Garneau était né à Québec,mais son père, tout comme celui de ce dernier, avait demeuré à Saint-Augustin. Le grandpère du jeune Garneau y habitait toujours d'ailleurs et, parfois, son petitfils se plaisait à l'y aller voir ${ }^{7}$. Petitclair dut y retourner souvent aussi, puisque c'est sur les rives du lac Saint-Augustin qu'il a plus tard situé l'action d'une Partie de campagne, sa dernière comédie. Mais ce ne sont pas là les seuls points que Garneau et Petitclair ont en commun : celui-ci marchera quelque temps sur les traces de celui-là, partagera avec lui un goût pour l'étude et pour les lettres, puis, comme lui, fera des voyages ${ }^{8}$.

A l'école, Petitclair se montra un élève brillant et il fit de rapides progrès. Dès le 8 octobre 1825, il entrait au Petit Séminaire de Québec, «en qualité d'externe, " affirme Darveau ${ }^{9}$, comme pensionnaire, découvre-t-on sur sa fiche d'écolier. Quoi qu'il en soit, là aussi il sut se distinguer par son intelligence et son application au travail, figurant parmi les premiers de sa classe et se méritant des éloges de ses professeurs. Le programme des cours du Séminaire était presque exclusivement axé sur l'enseignement du latin, mais il comprenait aussi la grammaire, l'histoire, la géographie et même la mythologie. À proprement parler, it n'y avait pas de cours de litté- 
rature, mais on étudiait les meilleurs auteurs classiques français. De plus, à la fin de l'année académique, les séminaristes récitaient des poèmes et jouaient des pièces devant leurs parents assemblés. C'est vraisemblablement à ce moment-là que Petitclair dut s'initier au théâtre ${ }^{10}$.

À l'automne de 1829, Petitclair entreprit sa Troisième année, mais à la fin du mois d'octobre, il dut, poussé par le besoin sans doute, quitter le Séminaire pour chercher du travail. Toutefois, il n'abandonna pas complètement les études. Employé comme commis au greffe judiciaire de Québec, il eut la chance d'acquérir quelques connaissances des lois grâce à un bienfaiteur, Joseph-François Perrault.

Ce personnage, dont il a déjà été question plus haut, était né à Québec le 2 juin 1753. Initié au commerce par son père, il ne tarda pas à abandonner les affaires pour s'intéresser au droit, devenir capitaine de milice et se faire élire député à la Chambre d'Assemblée. Après 1820, il se consacra surtout au notariat et à l'éducation. Partisan du système de Lancaster, il fonda des écoles laïques dans le faubourg Saint-louis, - dont l'une, justement, fut fréquentée par Garneau et Petitclair, - répandit l'enseignement mutuel et, vu la rareté des manuels scolaires à l'époque, écrivit divers traités de pédagogie, de géographie, de grammaire, d'histoire, de médecine vétérinaire et d'agriculture. II mourut en $1844{ }^{11}$.

Se rappelant sans doute l'intelligence du petit écolier, Maître Perrault offrit à Petitclair de lui servir en quelque sorte de précepteur. Pendant trois ans, assisté de son confrère, Edward Burroughs, il aida le jeune clerc à étendre la sphère de ses connaissances et, en bon érudit qu'il était, dut lui donner des leçons d'anglais, d'histoire et de philosophie, tout en essayant de l'intéresser au droit, comme il avait fait quelques années plus tôt avec F.-X. Garneau. Aussi n'est-ce pas un hasard si, à la fin de l'année 1832 ou au début de l'année 1833, Petitclair entra à l'étude d'Archibald Campbell chez qui le futur historien était devenu notaire en $1830^{12}$.

D'origine écossaise, Archibald Campbell était, tout comme J.-F. Perrault, un homme bon et généreux qui se plaisait à encourager les jeunes talents. Cachant une âme tendre sous des dehors revêches, il semble avoir poussé, avec ses clercs, la tolérance jusqu'à la complaisance. Telle est du moins la façon dont Petitclair le présente quand, dans la Donation, il le met en scène sous les traits du notaire Villomont. Obligé de faire tout le travail lui-même, celui-ci songe moins à réprimander ses clercs qu'à se moquer de leurs excentricités. II raille particulièrement l'un d'eux qui fait profession d'homme de lettres:

L'un est romanesque et littérateur, et, au lieu de lire les Institutes de Justinien ou la Coutume de Paris, il s'amusera à lire Jacob Faithful ou la Cuisinière canadienne. Il a aussi la manie de se croire poète, et, sans même savoir l'orthographe, il fait des vers à perte d vue: des alexandrins de dix-huit pieds, de vingt pieds, ça ne l'occupe pas. II va ensuite harceler les éditeurs, pour faire insérer sa production qu'il a la modestie de croire un chef-d'œuvre, et, après bien des 
démarches et en payant le prix d'une annonce, il parvient quelquefois à la faire insérer dans un journal... Grand Dieu! quelle gloire ${ }^{3}$ !

Nul doute que cette description caricaturale ne s'applique à Petitclair lui-même. Déjà, en 1830, il avait commencé à écrire des vers et il avait même réussi à en faire publier dans des journaux. De plus, c'était un liseur invétéré qui dévorait tout avec un égal appétit. Or le notaire Campbell possédait une bibliothèque bien garnie à laquelle ses employés avaient libre accès. Très cultivé lui-même, il faisait partie de la Société Historique de Québec, aimait les sciences et s'intéressait aux lettres ${ }^{14}$. On comprend que Petitclair se soit plu à travailler chez cet homme instruit et bienveillant. Simple copiste, il avait sans doute plus de loisirs que ses confrères pour parfaire ses connaissances et se livrer à ses occupations favorites.

En effet, peu tenté par le notariat, Petitclair abandonna l'étude du droit dès qu'il entra au service de Maître Campbell. Calligraphe accompli, il se contenta de grossoyer des actes et des contrats pour le compte de celui-ci, allant parfois au Parlement afin d'y copier les "bills" votés à la Chambre d'Assemblée. II se livra à cette occupation environ cinq ans semble-t-il, car on ne sache pas qu'il ait eu d'autres emplois durant son séjour à Québec, et il y demeura au moins jusqu'en 1837. On le sait par les quelques textes qu'il y fit publier durant ce temps: une romance, la Somnambule, en $1835^{15}$; un prospectus annonçant la publication d'une comédie-proverbe, Qui trop embrasse mal étreint, en $1836^{16}$; une farce, Griphon ou la vengeance d'un valet, en 1837'7, pour remplacer l'ouvrage précédent retenu au dernier moment; et une chanson folklorique, le Bon Parti, également en $1837^{18}$.

Dans la Somnambule, Petitclair met en scène une amante que torture le remords d'avoir trahi son amant. Ce thème de l'infidélité revient dans deux autres poèmes: $\grave{A}$ Flore et Sombre est mon âme comme vous. Cette façon d'envisager l'amour semble être le fruit d'une expérience personnelle Bien sûr, si l'on songe à Lamartine et à Chateaubriand qu'on lisait beaucoup au Canada à ce moment, on est tenté de croire à une passion plus littéraire que réelle. Cependant, on sait que Petitclair ne s'est jamais marié et, dans son théâtre, il est loin d'exalter l'amour. $A$ une exception près ${ }^{19}$, on n'y rencontre aucun couple heureux. Ainsi, dans la Donation, Caroline et Auguste avouent leurs sentiments réciproques à un tiers, mais placés l'un en face de l'autre, ils montrent une pudeur qui ressemble étrangement à de la honte. Dans Griphon et dans une Partie de campagne, l'amour est un piège dont on se sert pour berner, là un vieillard lubrique, ici un jeune ambitieux qui songe peut-être moins au bonheur qu'à sa situation sociale. Certes, Petitclair a très probablement voulu se moquer des mœurs qui prévalaient en son temps et qui faisaient de l'amour un «vice honteux ${ }^{20}$." Le clergé se montrait d'un rigorisme intransigeant sur ce sujet et, pour un auteur satirique, il y avait matière à s'amuser. Toutefois, il reste que l'attitude de Petitclair à l'égard de l'amour est assez constante pour paraître significative et il est certain qu'il est plus enclin à déprécier ce sentiment qu'à le valoriser. 
Ainsi donc, on peut en déduire que Petitclair a vécu une aventure sentimentale malheureuse. Le poème À Flore est une fable sur l'inconstance des amants et, vu que ce nom n'a aucune signification symbolique applicable dans ce cas-ci, il semble bien que ce titre soit aussi une dédicace. Quant à la romance Sombre est mon âme comme vous, Petitclair l'écrivit en 1830. II n'avait que dix-sept ans alors; à cet âge tendre, le cœur ne connaît pas de demi-mesures, ce qui doit expliquer pourquoi cette trahison l'a marqué d'une façon indélébile. Peu expansif par nature, il se replia davantage sur lui-même.

Petitclair quitta l'étude de Maître Campbell pour s'engager comme précepteur dans "une riche famille qui faisait en grand le commerce des pelleteries sur les côtes du Labrador 21." Avec sa discrétion habituelle, Darveau se garde bien de mentionner le nom de celle-ci. Par recoupement, on découvre une famille Labadie qui comptait douze enfants dont cinq ont prêté leurs noms à des personnages du théâtre de Petitclair. De plus, on remarque un détail particulier à propos de l'un de ces noms. Dans sa dernière pièce, une Partie de campagne, Petitclair met en scène un Canadien français anglomane, du nom de Guillaume, qui insiste pour se faire appeler William. Or l'aîné des Labadie, baptisé Guillaume, est prénommé William lors d'un recensement effectué en $1858^{22}$. Telle est donc la famille chez qui Petitclair devint précepteur.

A partir du moment où Petitclair entra chez les Labadie, son sort fut étroitement lié à celui de cette famille. II l'accoınpagna partout, ne retournant à Québec que pour de brefs séjours «tous les deux ou trois ans ${ }^{23}$." Si on suppose qu'il s'y rendit chaque fois que parurent de ses écrits. il y était en septembre-octobre 1839 (Sombre est mon âme comme vous ${ }^{24}$ ), en novembre 1840 (Une Aventure au Labrador ${ }^{25}$ ) et de novembre 1842 à janvier 1843 (Pauvre Șoldat! qu'il doit souffrir! ${ }^{26}$, À Flore ${ }^{27}$, La Donation ${ }^{28}$, Le Règne du Juste ${ }^{29}$ ). II semble même qu'il y passa tout l'hiver cette annéelà, car après une soirée musicale et dramatique au cours de laquelle avait été créée la Donation, le rédacteur de l'Artisan forma le vœu suivant :

Nous espérons que MM. Sauvageau et Petitclair se réuniront encore pour donner d'autres soirées durant le long hiver qui commence à nous envelopper dans son linceul de neige ${ }^{30}$.

La Donation fut reprise en octobre 1851 et en septembre 1858, mais rien n'indique que Petitclair était à Québec alors. Comme le texte en avait été publié dans l'Artisan en 1842 et dans le Répertoire national en 1848, et comme il n'existait pas de droit d'auteur à l'époque, des amateurs ont pu décider de la jouer sans même le prévenir. Il en fut de même pour une Partie de campagne. Petitclair était sans doute présent à sa création en 1857. Cependant, on remarque qu'il en avait alors confié la mise en scène à Joseph Savard, son parent et ami ${ }^{31}$. Or c'est ce même Joseph Savard qui fut responsable de la publication posthume de cette pièce en 1865 et de sa reprise en 1866. Comme il possédait le texte, il assura très probablement la reprise de 1860 également. Bien sûr, il n'est pas 
impossible que Petitclair ait assisté à l'une ou l'autre de toutes ces reprises, sauf celle de 1866 évidemment, mais il importe de savoir que celles-ci ne relèvent pas nécessairement de son initiative.

Si intéressant qu'il serait de savoir exactement quand Petitclair vint à Québec, il importerait davantage de connaître l'intinéraire qu'il suivit avec la famille Labadie. Malheureusement, à peine peut-on en déterminer quelques étapes à des remarques glanées çà et là. Dans son conte, une Aventure au Labrador, publié en novembre 1840, Petitclair glisse le détail suivant :

Je me trouvais, I'hiver dernier, à une de ces réunions joviales si fréquentes au Labrador dans la saison des neiges ${ }^{32}$.

De plus, c'est sous le pseudonyme Labrador qu'il fit paraître sa romance Sombre est mon âme comme vous à l'automne 1839. Lui et les Labadie sont donc demeurés quelque temps dans cette contrée. Où exactement? Or ne saurait le dire, mais il y a.fort à parier que c'est dans la région de Blanc-Sablon ${ }^{33}$ d'où était originaire Geneviève Goodchild, l'épouse de Guillaume-Louis Labadie, et où la famille finit par s'établir de façon définitive. Entretemps, ils allèrent habiter également en Gaspésie ${ }^{34}$. En 1858 , on retrouve les Labadie confortablement installés à l'Anse des Dunes, près de Blanc-Sablon. Selon un témoin, Placide Vigneau, la famille vivait dans l'aisance et connaissait la prospérité ${ }^{35}$. Malheureusement, le père mourut vers 1865 et les siens dilapidèrent ses biens très rapidement ${ }^{36}$.

Petitclair ne fut pas le témoin de la ruine des Labadie, car il mourut le 15 août 1860. Quelques jours avant sa mort, l'abbé François-Xavier Plamondon, effectuant une tournée missionnaire sur la Côte Nord, séjournait chez les Labadie. II raconte ainsi sa visite:

J'arrivai le 6 (août) chez Monsieur Labadie, à l'Anse des Dunes, près de Blanc-Sablon. Là se terminait ma longue mission. Il y a une petite chapelle bâtie depuis deux ans pour la commodité des familles environnantes. Il y a 9 familles à l'Anse des Dunes, la Longue Pointe et Blanc-Sablon. Durant six jours qu'ont duré les exercices, j'ai eu la consolation de communier 30 personnes, de faire faire trois premières communions, de baptiser huit enfants et de bénir deux mariages. Je terminais mes missions le onze août, et le lendemain, je prenais passage, pour mon retour à Québec, à bord de la goélette du Capitaine Nazaire Blais ${ }^{37}$.

Aucune mention n'est faite d'un malade ou d'un mourant. C'est pourquoi on est porté à croire que la mort de Petitclair a dû être soudaine. Par ailleurs, on sait par Joseph Savard que Petitclair est décédé non à l'Anse des Dunes, mais à Pointe au Pot ${ }^{38}$. Là demeurait Martin Parant, un beaufrère de Guillaume-Louis Labadie, qui, lui, avait deux enfants ${ }^{39}$. On peut supposer que Petitclair avait été chargé de leur instruction à eux aussi, ou bien qu'il était à Pointe au Pot pour affaires. En effet, vu qu'il possédait quelques notions de droit et qu'il n'y avait ni notaire ni avocat dans cette région reculée, il remplissait les fonctions d'homme de loi au besoin. Quoi qu'il en soit, il mourut là où il se plaisait à vivre, car il chérissait la soli- 
tude. Quelques mois après son décès, le Journal de l'instruction publique rapporta l'événement en lui rendant ce bref hommage:

M. Petitclair, auteur de plusieurs petites comédies canadiennes, qui ne manquaient ni de verve ni d'originalité, et de quelques pièces de vers qu'on trouve dans le Répertoire national de M. Huston, est mort il y a déjà plusieurs mois ${ }^{40}$.

Il était dans sa quarante-septième année.

Bien que ce soit là tout ce que l'on sache de la vie de Petitclair, on possède, toujours grâce à Darveau, assez de renseignements sur lui pour se faire une idée du genre d'homme qu'il était. Au physique :

Petitclair était de haute taille - ayant près de six pieds, - droit, musculeux et robuste, le front largement développé, teint brun, cheveux noirs et yeux gris mais sombres et perçants ${ }^{41}$.

Ce portrait, le seul que l'on ait de Petitclair, ne laisse guère deviner le trait marquant de sa personnalité. En effet, malgré son imposante stature, celui-ci était un homme timide à l'excès. D'un laconisme désespérant d'ordinaire, Darveau ne tarit pas sur ce point. Selon lui, Petitclair souffrait de ce défaut comme d'une maladie, et à un point tel que ce serait là la cause de tous ses déboires, de tous ses échecs et, finalement, de l'oubli dans lequel il a sombré. Il explique:

Ce côté faible de son caractère lui a toujours nui considérablement et l'a constamment tenu en arrière, à l'écart, dans l'oubli et dans l'obscurité, quand, avec un peu plus de hardiesse, plus d'ambition, ou plutôt plus de force, plus de volonté pour en avoir, il aurait infailliblement, avec les talents supérieurs dont il était si merveilleusement pourvu, il aurait, disons-nous, certainement brillé au premier rang et se serait fait une belle position sociale. II y avait chez lui de l'étoffe pour faire un de nos hommes les plus marquants et les plus distingués ${ }^{42}$.

Autrement dit, Petitclair avait tous les talents, sauf un: celui de les faire valoir.

Mais quels sont-ils, tous ces talents, dont Petitclair paraît avoir été doté si richement? Darveau les énumère et leur nombre est pour le moins impressionnant. Lecteur infatigable, Petitclair lisait tout, dévorait tout, en anglais comme en français, et son intérêt s'étendait aussi bien aux sciences qu'aux arts. Doué pour les mathématiques, il excellait plus particulièrement en géométrie. Épris de philosophie, il était un logicien remarquable: la fermeté de son jugement et la solidité de son raisonnement excitaient l'admiration de ses amis quand, ayant surmonté sa timidité, il se lançait dans des discussions avec eux. Mélomane accompli, il jouait de plusieurs instruments, dont la clarinette, le violon et la guitare, possédait "une magnifique voix de basse, que Lablache n'eût pas dédaignée $^{43}$, et s'exerçait même à la composition. C'est pourquoi la plupart des poèmes qu'il a écrits sont des romances ou des chansons. Habile à manier 
le pinceau, il s'adonnait à la peinture avec beaucoup d'adresse, ce qui explique qu'il était un calligraphe très apprécié. Enfin, “il avait le génie de la conception littéraire, le feu de la poésie l'inspirait ${ }^{44}$.

Une fois qu'on a lu les ouvrages de Petitclair, on se prend à douter de ce panégyrique et, quand on connaît les enflures de nos rhétoriciens au siècle dernier, on n'a pas de peine à voir combien il est exagéré. Néanmoins, il en ressort un trait évident, à savoir que Petitclair était un dilettante, c'est-à-dire un homme qui aimait s'initier aux choses de l'esprit pour son. plaisir et son enrichissement personnels. En effet, on ne mettra pas en doute qu'il aimait l'étude, qu'il cherchait à se renseigner par tous les moyens, qu'il s'intéressait aux sciences les plus diverses et qu'il possédait de nombreuses aptitudes. Du reste, quand on étudie les sources de ses comédies, on se rend compte qu'il a tout lu, de Molière à Scribe, son contemporain. On s'étonne même de son érudition, compte tenu du fait qu'en son temps les livres étaient rares et que les valeurs intellectuelles avaient peu de poids. Aussi Petitclair dut-il être un des hommes les mieux cultivés de son milieu. Esprit curieux, ouvert à toutes les formes de connaissance, il possédait sûrement les qualités requises chez un excellent précepteur. Cependant, si avide de connaissances qu'il fût, il donne l'impression de n'avoir rien approfondi. En d'autres mots, il se serait contenté de survoler les matières qui l'intéressaient plutôt que de les étudier d'une manière exhaustive.

Qu'on examine sa carrière littéraire, par exemple. Commencée vers 1830, 'elle démarre assez lentement, devient plus intense à partir de 1837, puis s'arrête brusquement au tout début de l'année 1843. Après cette date, Petitclair n'écrira plus qu'une pièce, une Partie de campagne, en 185645 . La courbe est bizarre: on dirait un intérêt passager, presque un caprice. De fait, Petitclair ne paraît pas avoir attaché beaucoup d'importance à ce qu'il écrivait, à son théâtre surtout. Sur les cinq pièces qu'il ait produites, deux, Qui trop embrasse mal étreint et le Brigand, sont restées inédites et demeurent introuvables. Des trois autres, seul Griphon a été publié par ses soins. Quant à la Donation et à une Partie de campagne, on l'a vu, c'est James Huston et Joseph Savard qui se sont occupés de l'une et de l'autre respectivement. De plus, en annonçant la publication de sa comédieproverbe à laquelle il a fini par substituer Griphon, Petitclair avouait :

Le soussigné ne se proposait pas, quand il a commencé son oeuvre, de la livrer à l'impression, (c'était pour lui un amusement,) ce n'est que pour se rendre à l'invitation de ses amis qu'il le fait ${ }^{46}$.

“C'était pour lui un amusement,» et telle est, en fin de compte, définie par Petitclair lui-même, sa manière de concevoir l'acte d'écrire. Dès lors, il convient de voir dans ses ouvrages les exercices d'un amateur qui n'avait nulle autre prétention que de se divertir en se livrant à des jeux intellectuels.

En somme, Petitclair avait de lui-même une opinion plus modeste que celle de Darveau. A la pensée qu'on lui eût cru du génie, 
il eût rougi de confusion sans doute, ou peut-être eût-il simplement ri, lui, notre premier auteur comique. Quoi qu'il en soit, il est bon de savoir qu'il n'entretenait aucune illusion quant à l'importance de son œuvre littéraire. Même si le jugement qu'il faut porter sur elle ne peut pas être atténué pour autant, on doit reconnaître que Petitclair était conscient de ses limites et de ses possibilités. Le but de la vie, affirmait Socrate, doit être la conquête de soi. Si Petitclair a reconnu quel était son mérite, il a fait un merveilleux pas en ce sens. Lui qui préférait la solitude et l'isolement dans la nature, lui qui se plaisait à méditer et à réfléchir, sans doute mettait-il plus de prix à s'accomplir comme homme qu'à briller comme écrivain.

1. Louis-Michel Darveau, Petitclair, in Nos Hommes de lettres, Montréal, A.-A. Stevenson, 1873, p. 61-74. Dans cette esquisse biographique, nous indiquons ces inexactitudes.

2. Et non en août 1813, comme l'indique Darveau, in idem, p. 61. Par ailleurs, on peut se demander pourquoi Alain Pontaut se contente d'être approximatif en indiquant simplement "né aux alentours de 1810 " dans son Dictionnaire critique du théatre québécois, Montréal, Leméac, 1972, p. 122.

3. Extrait du Registre des baptêmes, mariages et sépultures, paroisse de SaintAugustin, comté de Portneuf, Québec.

4. Ces renseignements nous ont été gracieusement fournis par l'Institut généalogique Drouin de Montréal.

5. Extrait du Registre des baptêmes, mariages et sépultures, paroisse NotreDame de I'Annonciation, Ancienné Lorette, Québec.

6. Voir Jean-Jacques Jolois, Joseph-François Perrault (1753-1844) et les origines de l'enseignement laïque au Bas-Canada, Montréal, P.U.M., 1969, p. 221-230.

7. Voir Gustave Lanctot, François-Xavier Garneau, Toronto, The Ryerson Press, 1926 , p. 3-6.

8. Voir Victor Morin, Un Pionnier du theâtre: Pierre Petitclair, in La Revue moderne, Montréal, déc. 1932, $14^{e}$ année, no 2, p. 6.

9. L.-M. Darveau, op. cit., p. 62.

10. Voir Honorius Provost, Le Séminaire de Québec - documents et biographies, Québec, Publications des Archives du Séminaire de Québec, 1964, p. 302303 ; et Pierre Savard, L'Enseignement de l'histoire et de la géographie, in Aspects de l'enseignement au Petit Séminaire de Québec 1765-1945, Québec, La Société historique de Québec, Cahiers d'histoire no 20, 1968, p. 87-102.

11. Voir J.-J. Jolois, op. cit., p. 18-20 et 77-181.

12. Voir G. Lanctot, op. cit., p. 8-11.

13. Pierre Petitclair, La Donation, acte II, scène 20.

14. Voir G, Lanctot, op. cit., p. 8-9.

15. Nous n'avons pas trouvé cette romance dans les journaux de 1835 , mais elle fut recueillie par James Huston dans le Répertoire national, 1ère éd., Montréal, Lovell et Gibson, 1848, vol. I, p. 278; 2 éd., Montréal; J.-M. Valois \& Cie, 1893, vol. I, p. 322.

16. Pierre Petitclair, Comédie - Prospectus (Qui trop embrasse mal étreint), in Le Canadien, Québec, 7 nov. 1836, vol. VI, no 78, p. 2.

17. Idem, Griphon ou la vengeance d'un valet, Québec, William Cowan, 1837, $90 \mathrm{p}$.

18. Idem, Le Bon Parti, in Le Télégraphe, Québec, 24 mars 1837, vol. 1, no 3, p. 1.

19. Le couple Flore et Baptiste dans une Partie de campagne.

20. Pierre Petitclair, Griphon ou la vengeance d'un valet, acte I, scène 5 . 
21. L.-M. Darveau, op. cit., p. 63.

22. Registre des Missions de la Côte du Labrador, Recensement de 1858, Archives de l'Évêché de Hauterive.

23. L.-M. Darveau, op. cit., p. 63.

24. Pierre Petitclair, Sombre est mon âme comme vous, in Le Fantasque, Québec, 1 er oct. 1839 , vol. II, no 11, p. 81.

25. Idem, Une Aventure au Labrador, in idem, 2 et 9 nov. 1840, vol. 2, nos 46 et 47 , p. 361-363 et 369-374.

26. Idem, Pauvre Soldat! qu'il doit souffrir!, in L'Artisan, Québec, 28 nov. 1842 , vol. I, no 15, p. 1.

27. Idem, A Flore, in idem, 12 déc. 1842, vol. I, no 19, p. 1.

28. Idem, La Donation, in idem, 15, 19, 2226 et 29 déc. 1842, vol. I, nos 20-24.

29. Idem, Le Règne du Juste, in idem, 2 janv. 1843, vol. I, no 25, p. 1 ; Le Canadien, Québec, 4 janv. 1843, vol. XII, no 102, p. 2 ; et Le Journal de Québec, Québec, 10 janv. 1843, 1ère année, no 12, p. 2.

30. Voir Seconde Soirée musicale de Mr. Sauvageau (sic), in L'Artisan, Québec, 21 nov. 1842, vol. I, no 13, p. 2.

31. Voir Un Spectateur, Les Amateurs canadiens-français, in Le Canadien, Québec, 27 avril 1857, vol. 26, no 151, p. 2. Joseph Savard avait comme grand-mère paternelle Marie-Anne Bussière, sœur de Magdeleine Bussière qui, elle, était grand-mère maternelle de Petitclair. Ils étaient donc cousins. Par ailleurs, nous pensons que Joseph Savard aurait épousé Marie-Cécile, la sœur de Petitclair.

32. Pierre Petitclair, Une Aventure au Labrador, in op. cit., p. 362.

33. Blanc-Sablon est situé à un mille à peine de la frontière qui sépare le Québec du Labrador, sur la rive nord du Saint-Laurent.

34. Voir J. Huston, op. cit., 1ère éd., p. 278.

35. Placide Vigneau, Notes historiques sur la Côte Nord, copie miméographique, Havre Saint-Pierre, 1900.

36. Voir Pierre-Étienne Fortin, Rapport annuel de Pierre Fortin, Écuier, magistrat stipendiaire, commandant l'expédition pour la protection des pêcheries dans le golfe du Saint-Laurent, à bord de "La Canadienne" pendant la saison de 1866, in Canada, Assemblée législative, Rapport du commissaire des terres de la Couronne, (s. 1.), (s. é.), 1866, p. 90-121.

37. Rapports sur les missions du diocèse de Québec, 1839-1874, Saint-Roch de Québec.

38. Joseph Savard, Préface, in Pierre Petitclair, Une Partie de campagne, Québec, J. Savard, 1865, p. 5.

39. Ils avaient épousé les deux sœurs: G.-L. Labadie, Geneviève Goodchild, et M. Parant, Mary Goodchild. Voir le Registre des missions de la Côte du Labrador, Recensement de 1858, Archives de l'Évêché de Hauterive.

40. Journal de linstruction publique, Montréal, déc.1 1860, vol. IV, no 12, p. 208.

41. L.-M. Darveau, op. cit., p. 73-74.

42. Ibidem, p. 66 .

43. Ibidem, p. 65. Louis Lablache (1794-1858) a été reconnu universellement comme une basse admirable.

44. Ibidem, p. 66.

45. Et peut-être aussi le Brigand dont on ignore la date de composition.

46. Pierre Petitclair, Comédie - Prospectus (Qui trop embrasse mal étreint), in op. cit., p. 2. 